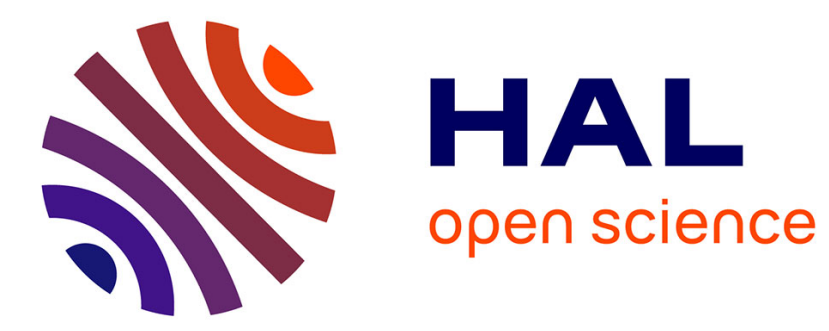

\title{
Optical fiber analogous of the piston shock problem
}

Abdelkrim Bendahmane, Gang Xu, Matteo Conforti, Alexandre Kudlinski, Arnaud Mussot, Stefano Trillo

\section{To cite this version:}

Abdelkrim Bendahmane, Gang Xu, Matteo Conforti, Alexandre Kudlinski, Arnaud Mussot, et al.. Optical fiber analogous of the piston shock problem. Nonlinear Photonics, Jul 2018, Zurich, Switzerland. pp.JTu6G.2, 10.1364/BGPPM.2018.JTu6G.2 . hal-02393187

\section{HAL Id: hal-02393187 https://hal.science/hal-02393187}

Submitted on 6 Dec 2019

HAL is a multi-disciplinary open access archive for the deposit and dissemination of scientific research documents, whether they are published or not. The documents may come from teaching and research institutions in France or abroad, or from public or private research centers.
L'archive ouverte pluridisciplinaire HAL, est destinée au dépôt et à la diffusion de documents scientifiques de niveau recherche, publiés ou non, émanant des établissements d'enseignement et de recherche français ou étrangers, des laboratoires publics ou privés. 


\title{
Optical fiber analogous of the piston shock problem
}

\author{
Abdelkrim Bendahmane ${ }^{1, *}$, Gang $\mathrm{Xu}^{1}{ }^{1, *}$, Matteo Conforti ${ }^{1}$, Alexandre Kudlinski ${ }^{1}$, Arnaud Mussot ${ }^{1}$, \\ Sefano Trillo ${ }^{2}$ \\ ${ }^{1}$ Univ. Lille, CNRS, UMR 8523-PhLAM-Physique des Lasers Atomes et Mol'ecules, F-59000 Lille, France \\ ${ }^{2}$ Department of Engineering, University of Ferrara, Via Saragat 1, 44122 Ferrara, Italy \\ *These authors contributed equally to this work \\ abdelkrim.bendahmane@phlam.univ-lille1.fr
}

\begin{abstract}
We investigate the optical analogous of the piston shock problem in gas dynamics. Using fast temporal measurements, we recorded dispersive shock waves formed by the propagation of a bi-chromatic photon fluid along an optical fiber. $\odot 2018$ The Author(s)

OCIS codes: (190.4370) Nonlinear optics fibers, (190.5530) Pulse propagation and temporal solitons.
\end{abstract}

\section{Introduction}

Shock waves have been thoroughly investigated during the last century in many different branches of physics [1]. In conservative systems, shock waves are regularized by the formation, owing to dispersion, of rapidly oscillating nonstationary structures, called dispersive shock waves (DSWs). DSWs are now the subject of intense theoretical and experimental studies in many physics areas like Bose-Einstein condensates [2] or unitary Fermi gases [3].

In nonlinear optical fibers, recent experimental studies of the generation of DSWs in the context of wave propagation with discontinuities i.e. the Riemann problems attracted growing attentions [4]. Here, we extend this subject by studying the case of the discontinuity of velocity inside a propagating pulse. This scenario is analogous to the classical piston shock problem where a gas is compressed by a moving piston [5].

\section{Analogy with the piston problem and experimental setup}

More precisely, we investigate the propagation, along a highly normal dispersive optical fiber, of a chirped square pulse with an abrupt jump of instant frequency (velocity) at its center (as illustrated in Fig. 1(a)). During the propagation of this dual-frequency pulse, the two parts propagate at different velocities mimicking gas compression. Indeed, half of the pulse (the fast part) plays the role of a moving piston while the other half (the slow part) plays the role of the compressed gas. The internal collision and squeezing of these two parts eventually lead to the generation of a plateau located in the center of the pulse, as well as a pair of DSWs aside of this plateau (Fig. 1(b)).

In order to generate the suitable bi-velocity square pulse needed to study the genuine Riemann problem, we temporally couple a square shaped pulse and a strong external M-shaped pulse. Both of these pulses are obtained by a direct intensity modulation of CW waves (Fig. 1(c)). The role of this M-shaped pulse is to induce a frequency shift on the square pulse via cross phase modulation (XPM) while propagating along $500 \mathrm{~m}$ of highly nonlinear fiber (HNLF). Since the linear slope of the M-shaped pulse abruptly change at its center, this allows to induce a quick shift in frequency at the center of the square pulse. Hence, synthesizing the desired state.

At the output of the HNLF fiber, the M-shaped pulse is spectrally filtered and the chirped square pulse is then injected into $15-\mathrm{Km}$ of dispersion compensating fiber (DCF) (fiber parameters are given in the caption of Fig. 1). The output light is characterized using an optical sampling oscilloscope (OSO).
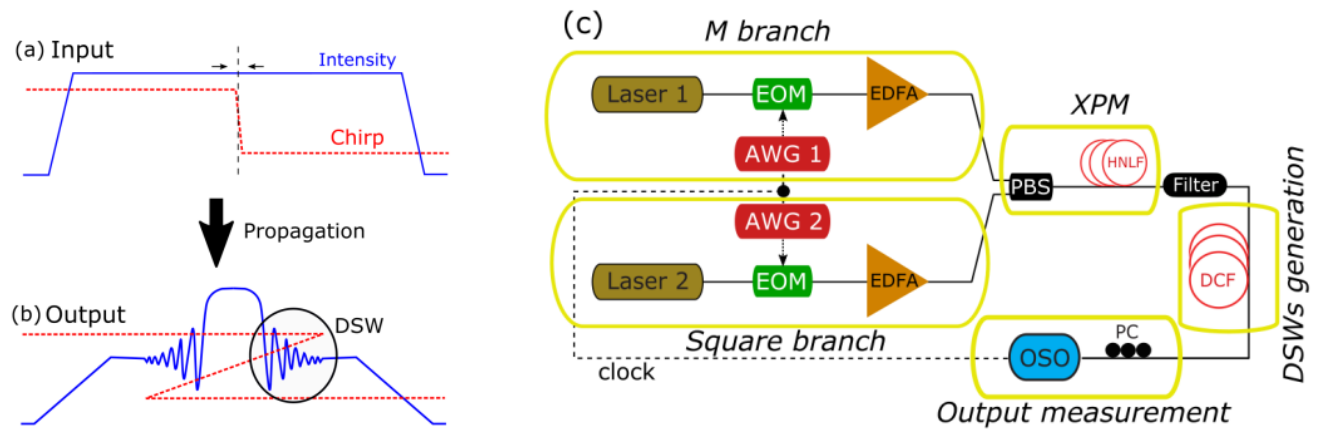

Fig. 1. (a,b) Illustration of the chirped square pulse and the generation of DSWs. (c) Experimental setup. AWG, Arbitrary Waveform Generator; EOM, electro-optic modulator; EDFA, erbium-doped fiber amplifier; PBS, polarization beam splitter; PC, Polarization controller; OSO, optical sampling oscilloscope; HNLF (length $500 \mathrm{~m}$; loss $\alpha=0.6 \mathrm{~dB} / \mathrm{km}$; zero dispersion wavelength ZDW $=1551.4 \mathrm{~nm}$; third order dispersion $\beta_{3}=0.53 \times 10^{-40} \mathrm{~s} / \mathrm{m}$; nonlinear coefficient $\left.\gamma=12 \mathrm{~W}^{-1} \mathrm{~km}^{-1}\right)$. DCF(length $15 \mathrm{~km} ; \alpha=0.5 \mathrm{~dB} / \mathrm{km} ; \beta_{2}=1.7 \times 10^{-25} \mathrm{~s}^{2} / \mathrm{m} ; \gamma=3 \mathrm{~W}^{-1} \mathrm{~km}^{-1}$ ); Initial condition of M pulse (peak power $7 \mathrm{~W}$, duration $2.4 \mathrm{~ns}$, @ $1541 \mathrm{~nm}$ ) and square pulse (peak power $70 \mathrm{~mW}$, duration $1.6 \mathrm{~ns}$, @ $1545 \mathrm{~nm}$ ). 


\section{Piston configuration: compression case}

We performed a series of experiments that demonstrate the generation of dispersive shock waves from the chirped pulse. Figure 2 (a) depicts the temporal profile of the square shaped pulse recorded at the DCF input (HNLF output). It has a duration of $1.6 \mathrm{~ns}$ and a peak power of $100 \mathrm{~mW}$. The spectrogram in Fig. 2(b) provides us a clear description of the bi-chromatic properties of this pulse. This spectrogram was obtained by performing a heterodyne detection with the help of a tunable $\mathrm{CW}$ laser and a $70 \mathrm{GHz}$ photodiode. In the presented case, the total frequency shift induced by the M-shaped pump is $11.5 \mathrm{GHz}$.

Once injected into the DCF fiber, the square pulse undergoes nonlinear effect and we observed at the DCF output, the generation of a short plateau in the center of the pulse, with several fast and regular oscillations aside (Fig. 2(c)). These oscillations correspond to a regularization of the shock and the formation of a pair of DSW [2].

The propagation of the chirped square pulse in the DCF was also numerically simulated by integrating the generalized nonlinear Schrodinger equation. Our simulations take into account second order of dispersion, linear losses, fiber nonlinearity and the Raman effect. Figure 2(d) depicts the simulated output profile corresponding to our experimental parameters. We observe the formation of a pair of DSW connected by a short plateau in a good qualitative agreement with observations. Note that the losses of the DCF has very slight impact on the DSWs dynamics changing neither the global form of the shock pair nor the plateau between them.
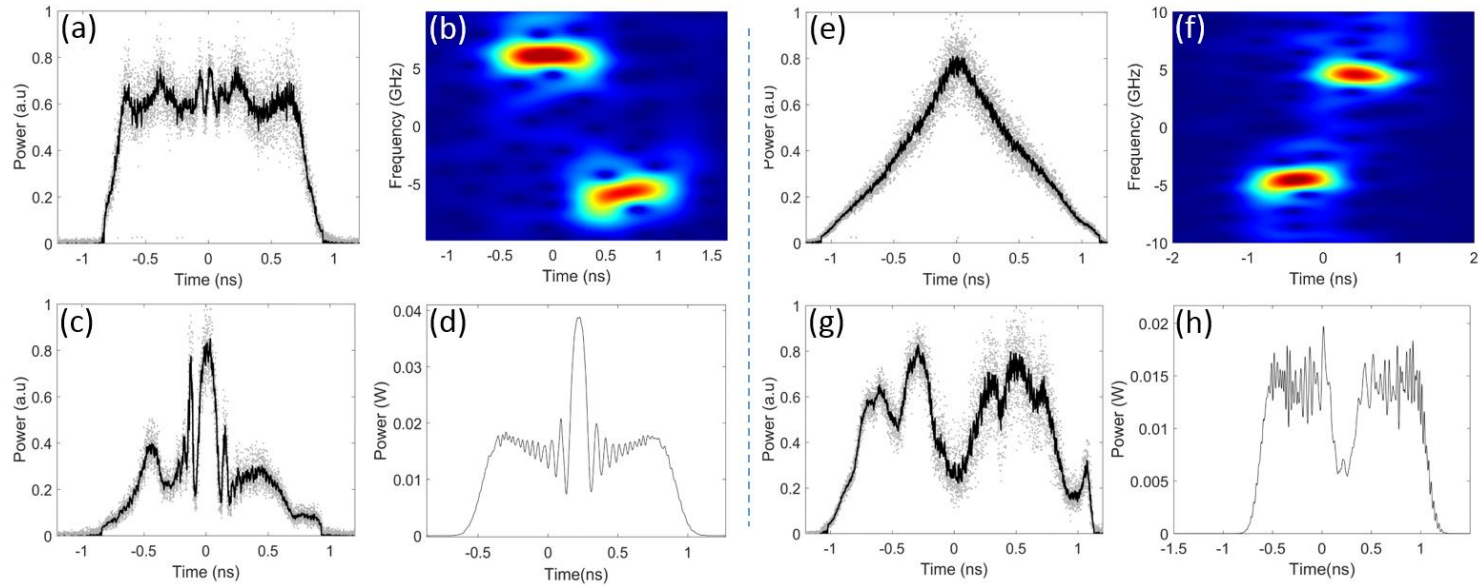

Fig. 2. (a-d) Piston configuration. (a) Intensity profile of the square pulse recorded at the DCF input. (b) Associated Spectrogram. (c) Intensity profile recorded at the DCF output. (c) Simulated Intensity profile at the DCF output. (e-h) Antipiston configuration. (e) Intensity profile of the triangle shaped pulse used to induce the chirp. Initial condition of the triangle pulse (peak power $5 \mathrm{~W}$, duration $2.4 \mathrm{~ns}$, @ $1536 \mathrm{~nm}$ ). (f) Spectrogram associated to the input square pulse (induced frequency shift $=9.1 \mathrm{GHz}$ ). (g) Intensity profile recorded at the DCF output. (h) Simulated intensity profile at the DCF output.

\section{Anti-piston configuration: decompression case}

In a second set of experiments, we replaced the M-shaped pump by a triangle shaped one (Fig. 2(e)), which reverses the sign of the velocity profile compared to the previous case (see spectrogram in Fig. 2(f)). Instead of compressing each other, the two parts composing the pulse undergo a decompression mechanism leading to the formation of a rarefaction waves pair, connected by a finite plateau (as represented in Fig. 2(g)). This process is analogous to the gas dynamics case when the piston decompresses and exhausts the gas, leading to a low pressure inside a reservoir. Here also, numerical simulations agree well with experiments (Fig. 2(h)).

\section{Conclusion}

We studied the optical analogy of a gas compressed/decompressed by a piston. Our experiments consist in the propagation of a bi-chromatic photon fluid along a highly dispersive optical fiber. We observed generation of dispersive shock waves and rarefaction waves. These results are also well supported by numerical simulations and pave the way to study gas dynamics in relatively accessible optical fiber experiments.

\section{References}

[1] G.B. Whitham, Linear and nonlinear waves (Wiley, New York, 1974).

[2] Z. Dutton et al., "Observation of quantum shock waves created with ultra-compressed slow light pulses in a bose-einstein condensate".

Science, 293, 663, (2001).

[3] J. Joseph et al., "Observation of shock waves in a strongly interacting fermi gas". Phys. Rev. Lett., 106, 150401(2011).

[4] G. Xu et al. "Dispersive dam-break flow of photon fluid", Phys. Rev. Lett., 118,254101(2017).

[5] M. Hoefer et al., "Piston Dispersive shock wave problem", Physical Review Letters, 100, 084504(2008). 